\title{
GENERATORS FOR VECTOR BUNDLES ON GENERIC HYPERSURFACES
}

\author{
N. Mohan Kumar, A. P. RaO, and G. V. Ravindra \\ AbStRact. We prove that on a generic hypersurface in $\mathbb{P}^{m+1}$ of dimension at least 3 , \\ a vector bundle with $r \leq m$ generators must be split if $m$ is odd. If $m$ is even, then the \\ same is true if the degree of $X$ is at least 3 .
}

\section{Introduction}

Let $\left(V, \mathcal{O}_{V}(1)\right)$ be a smooth polarized projective variety and $E$ be a vector bundle on $V$. We say $E$ is $r$-generated if there is a surjection $\bigoplus_{i=1}^{r} \mathcal{O}_{V}\left(a_{i}\right) \rightarrow E, a_{i} \in \mathbb{Z}$. The aim of this note is to extend to hypersurfaces, the following splitting criterion for vector bundles on projective space due to Faltings.

Theorem 1 ([2], Satz 1). A vector bundle on $\mathbb{P}^{m}$ is split if it can be generated by $r \leq m$ sections.

Based on an idea in [7], we prove the following.

Theorem 2. Let $E$ be any $r$-generated rank $n$ vector bundle on a smooth hypersurface $X$ of dimension $m \geq 3$. Then $E$ is a sum of line bundles if

(1) $r \leq m-1$.

(2) $r=m$ and $n \neq m / 2$.

(3) $X$ is generic of degree $d \geq 3, r=m$ and $n=m / 2$.

The first two parts of the theorem have proofs similar to Faltings' with the additional use of the Weak Lefschetz theorem. The third part is the main result of this paper and the outline of its proof is as follows. In the first section, following [7], we introduce a certain thickening of $X$ in its universal family which we call $X_{\epsilon}$ and show that images of the cohomology of the projective space and $X_{\epsilon}$ in the cohomology of $X$ coincide (Corollary 1 ). We then show that if a generic hypersurface has a vector bundle as in the statement of the theorem, then this implies that there is such a bundle on $X_{\epsilon}$ (section 3). Then using certain standard arguments involving Chern classes, we conclude that such a bundle must be split. We remark that alternatively one could use the standard Noether-Lefschetz theorem on Hodge classes to prove the desired theorem. However the use of $X_{\epsilon}$ makes the proof simple and self-contained.

The result is sharp for low values of $m$; for quadrics in $\mathbb{P}^{5}$ and $\mathbb{P}^{7}$, we can find 4-generated rank 2 bundles and 6 -generated rank 3 bundles respectively. Since these examples can be pulled back via finite morphisms $\mathbb{P}^{l} \rightarrow \mathbb{P}^{l}$ (with $l=5,7$ ), we get

Received by the editors October 15, 2005.

1991 Mathematics Subject Classification. 14F05.

Key words and phrases. Vector bundles, Chern classes, Hodge cohomology. 
other examples on various smooth hypersurfaces. Hence the requirement of genericity is needed. We do not know any examples on quadrics or special smooth hypersurfaces in dimensions greater than six.

1.1. Conventions. We work over $k=\mathbb{C}$, the field of complex numbers. By a generic point of a variety, we mean a point outside a countable union of proper closed subvarieties.

\section{Cohomology computations}

2.1. Preliminaries. Let $\mathbb{P}:=\mathbb{P}_{k}^{m+1} S:=\mathbb{P}\left(W^{*}\right)$ where $W=\mathrm{H}^{0}\left(\mathbb{P}, \mathcal{O}_{\mathbb{P}}(d)\right)$ denotes the parameter space of all degree $d$ hypersurfaces in $\mathbb{P}$. One has a short exact sequence

$$
0 \rightarrow \mathcal{V} \rightarrow \mathrm{H}^{0}\left(\mathbb{P}, \mathcal{O}_{\mathbb{P}}(d)\right) \otimes \mathcal{O}_{\mathbb{P}} \rightarrow \mathcal{O}_{\mathbb{P}}(d) \rightarrow 0 .
$$

Let $\mathcal{X}:=\mathbb{P}\left(\mathcal{V}^{*}\right) \rightarrow S$ be the universal family of all degree $d$ hypersurfaces. Let $X \subset \mathbb{P}$ be a smooth degree $d$ hypersurface corresponding to a closed point $s \in S$. Let $V:=$ $T_{S, s}^{*}$, the dual of the Zariski tangent space at the point $s \in S . A:=\mathcal{O}_{S, s} / \mathfrak{m}_{s}^{2}=k \oplus V^{*}$ then over the subscheme $\operatorname{Spec} A \subset S$, there is a hypersurface which we will denote by $X_{\epsilon}$, the (infinitesimal) universal hypersurface over $\operatorname{Spec} A$. It is easy to see that $\Omega_{A}^{1} \otimes k \cong V^{*}$.

Let $p: \mathbb{P}_{A} \rightarrow \mathbb{P}, q: \mathbb{P}_{A} \rightarrow \operatorname{Spec} A$ denote the two projections. Consider the cotangent sheaf sequence for the inclusion $\iota_{\epsilon}: X_{\epsilon} \hookrightarrow \mathbb{P}_{A}$,

$$
\mathcal{O}_{X_{\epsilon}}(-d) \rightarrow \Omega_{\mathbb{P}_{A}}^{1} \otimes \mathcal{O}_{X_{\epsilon}}=p^{*} \Omega_{\mathbb{P}}^{1} \otimes \mathcal{O}_{X_{\epsilon}} \oplus q^{*} \Omega_{A}^{1} \otimes \mathcal{O}_{X_{\epsilon}} \rightarrow \Omega_{X_{\epsilon}}^{1} \rightarrow 0 .
$$

On restricting this sequence to $X$, we have

$$
0 \rightarrow \mathcal{O}_{X}(-d) \stackrel{(\alpha, \beta)}{\longrightarrow} \Omega_{\mathbb{P}}^{1} \otimes \mathcal{O}_{X} \oplus V^{*} \otimes \mathcal{O}_{X} \stackrel{(\gamma, \delta)}{\longrightarrow} \Omega_{X_{\epsilon}}^{1} \otimes \mathcal{O}_{X} \rightarrow 0 .
$$

Let $F$ denote the polynomial defining $X_{\epsilon} \subset \mathbb{P}_{A}$. On taking cohomology of the sequence $0 \rightarrow \mathcal{O}_{\mathbb{P}} \rightarrow \mathcal{O}_{\mathbb{P}}(d) \rightarrow \mathcal{O}_{X}(d) \rightarrow 0$, we get $0 \rightarrow k \rightarrow W \rightarrow V \rightarrow 0$. Choose a splitting $\theta: V \rightarrow W$, so that $\theta \in W \otimes V^{*}$. Since $W \otimes A=W \oplus W \otimes V^{*}$, we have $F=(f, \theta) \in W \otimes A=\mathrm{H}^{0}\left(\mathbb{P}_{A}, \mathcal{O}_{\mathbb{P}_{A}}(d)\right)$ where $f$ is the polynomial defining $X$. The map $d F$ is then given by $(\tilde{\alpha}, \tilde{\beta})$. It is easy to see that $\tilde{\alpha}_{\left.\right|_{X}}=\alpha=d f$. By local computations, we can verify that $\tilde{\beta}_{\left.\right|_{X}}=\beta: \mathcal{O}_{X}(-d) \rightarrow V^{*} \otimes \mathcal{O}_{X}$ is dual to the evaluation map. Notice that the injectivity of $\delta$ follows from the injectivity of $\alpha$. We can also identify $\delta$ with the natural map obtained as follows. We have an exact sequence $q^{*} \Omega_{A}^{1} \rightarrow \Omega_{X_{\epsilon}}^{1} \rightarrow \Omega_{X_{\epsilon} / A}^{1} \rightarrow 0$, which is the relative cotangent sheaf sequence of the family $q: X_{\epsilon} \rightarrow \operatorname{Spec} A$. On restricting this sequence to $X$, we get $0 \rightarrow q^{*} \Omega_{A}^{1} \otimes \mathcal{O}_{X} \stackrel{\delta}{\rightarrow} \Omega_{X_{\epsilon}}^{1} \otimes \mathcal{O}_{X} \rightarrow \Omega_{X_{\epsilon} / A}^{1} \otimes \mathcal{O}_{X}=\Omega_{X}^{1} \rightarrow 0$.

The exactness of sequence (2.1) implies that $\gamma \alpha=-\delta \beta$ and as a consequence, we have the following

Lemma 1 (Mohan Kumar-Srinivas, [7]). There is a commutative diagram:

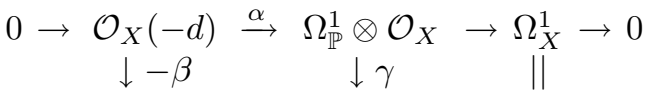

$$
\begin{aligned}
& 0 \rightarrow V^{*} \otimes \mathcal{O}_{X} \stackrel{\delta}{\rightarrow} \Omega_{X_{\epsilon}}^{1} \otimes \mathcal{O}_{X} \rightarrow \Omega_{X}^{1} \rightarrow 0
\end{aligned}
$$

where the two rows come from the inclusions $X \subset \mathbb{P}$ and $X \subset X_{\epsilon}$ and the map $\beta$ is the natural map. 
Taking the $\ell$-th exterior power, we get a commutative diagram

$$
\begin{aligned}
& 0 \rightarrow \Omega_{X}^{\ell-1}(-d) \rightarrow \Omega_{\mathbb{P}}^{\ell} \otimes \mathcal{O}_{X} \rightarrow \Omega_{X}^{\ell} \rightarrow 0 \\
& 0 \rightarrow \Omega(\ell) \rightarrow \Omega_{X_{\epsilon}}^{\ell} \otimes \mathcal{O}_{X} \rightarrow \Omega_{X}^{\ell} \rightarrow 0
\end{aligned}
$$

where $\Omega(\ell)$ (see [3], $5.16(\mathrm{~d})$, page 126) comes equipped with a decreasing filtration $\mathrm{F}^{*} \Omega(\ell)$ satisfying the properties $\mathrm{F}^{1}(\Omega(\ell))=\Omega(\ell), \mathrm{F}^{\ell+1}(\Omega(\ell))=0$ and $\operatorname{gr}_{\mathrm{F}}^{j} \Omega(\ell):=$ $\mathrm{F}^{j}(\Omega(\ell)) / \mathrm{F}^{j+1}(\Omega(\ell))=\Lambda^{j} V^{*} \otimes \Omega_{X}^{\ell-j}$ for $j \geq 1$.

\subsection{Odd dimensional hypersurfaces.}

Lemma 2. Let $X \subset \mathbb{P}^{m+1}$ be a smooth hypersurface and let $m=2 n+1$. Then

$$
\mathrm{H}^{p}\left(\mathbb{P}, \Omega_{\mathbb{P}}^{p}\right) \cong \mathrm{H}^{p}\left(X, \Omega_{X}^{p}\right) \quad \text { for } 0 \leq p \leq \operatorname{dim} X
$$

Proof. We first prove the statement for $0 \leq p \leq n$. By the Weak Lefschetz theorem, we have

For $i=2 p$, this implies that

$$
\mathrm{H}^{i}(\mathbb{P}, \mathbb{C}) \cong \mathrm{H}^{i}(X, \mathbb{C}) \quad \forall i \leq 2 n
$$

$$
\mathrm{H}^{p}\left(\mathbb{P}, \Omega_{\mathbb{P}}^{p}\right) \cong \mathrm{H}^{p}\left(X, \Omega_{X}^{p}\right) 0 \leq p \leq n
$$

For $n+1 \leq p \leq \operatorname{dim} X$, we proceed as follows: Using Serre duality, we have

$$
\mathrm{H}^{m-p}\left(X, \Omega_{X}^{m-p}\right) \cong \mathrm{H}^{p}\left(X, \Omega_{X}^{p}\right)^{\vee} \cong k \forall p
$$

Thus $h^{p}\left(X, \Omega_{X}^{p}\right)=1$ for $n+1 \leq p \leq \operatorname{dim} X$. Since the restriction map $\mathrm{H}^{p}\left(\mathbb{P}, \Omega_{\mathbb{P}}^{p}\right) \rightarrow$ $\mathrm{H}^{p}\left(X, \Omega_{X}^{p}\right)$ is non-zero, this implies that this map is an isomorphism for $n+1 \leq p \leq$ $2 n+1$. Thus we are done.

\subsection{Even dimensional hypersurfaces.}

Lemma 3. Let $X \subset \mathbb{P}^{m+1}$ be a smooth hypersurface with $m=2 n$. Then, $\mathrm{H}^{n}(X, \Omega(n))$ $=0$ where we recall that $\Omega(n)$ is the kernel of the map $\Omega_{X_{\epsilon}}^{n} \otimes \mathcal{O}_{X} \rightarrow \Omega_{X}^{n}$.

Proof. The proof follows by analysing the sequences

$$
0 \rightarrow \mathrm{F}^{j+1}(\Omega(n)) \rightarrow \mathrm{F}^{j}(\Omega(n)) \rightarrow \operatorname{gr}_{\mathrm{F}}^{j} \Omega=\Lambda^{j} V^{*} \otimes \Omega_{X}^{n-j} \rightarrow 0
$$

For $j \geq 1$, we first claim that $\mathrm{H}^{n}\left(X, \Omega_{X}^{n-j}\right)=0$. By the Weak Lefschetz theorem, $\mathrm{H}^{i}(\mathbb{P}, \mathbb{C}) \cong \mathrm{H}^{i}(X, \mathbb{C})$ for $i<\operatorname{dim} X$. Since $2 n-j<2 n, \mathrm{H}^{2 n-j}(X, \mathbb{C})$ is either $0(j$ odd) or one dimensional and isomorphic to $\mathrm{H}^{n-\ell}\left(X, \Omega_{X}^{n-\ell}\right)$ where $j=2 \ell$. Thus on taking cohomology, we have a surjection

$$
\mathrm{H}^{n}\left(X, \mathrm{~F}^{j+1}(\Omega(n))\right) \rightarrow \mathrm{H}^{n}\left(X, \mathrm{~F}^{j}(\Omega(n))\right) \rightarrow 0 \forall j \geq 1
$$

Since $\mathrm{H}^{n}\left(X, \mathrm{~F}^{n} \Omega(n)\right)=\mathrm{H}^{n}\left(X, \Lambda^{n} V^{*} \otimes \mathcal{O}_{X}\right)=0$, we are done.

Lemma 4. Let $X \subset \mathbb{P}^{m+1}$ be a smooth hypersurface with $m=2 n$. Then there are isomorphisms

$$
\begin{aligned}
& \mathrm{H}^{n+1}\left(X, \Omega_{X}^{n-1}(-d)\right) \cong \mathrm{H}^{2 n}\left(X, \mathcal{O}_{X}(-n d)\right) \\
& \mathrm{H}^{n+1}\left(X, \Omega_{X}^{n-1}\right) \cong \mathrm{H}^{2 n}\left(X, \mathcal{O}_{X}((-n+1) d)\right.
\end{aligned}
$$


Proof. We consider the exterior powers of the cotangent bundle sequence for the inclusion $X \subset \mathbb{P}$

$$
0 \rightarrow \Omega_{X}^{\ell-1}(-d) \rightarrow \Omega_{\mathbb{P}}^{\ell} \otimes \mathcal{O}_{X} \rightarrow \Omega_{X}^{\ell} \rightarrow 0
$$

together with the sequences

$$
0 \rightarrow \Omega_{\mathbb{P}}^{\ell}(-d) \rightarrow \Omega_{\mathbb{P}}^{\ell} \rightarrow \Omega_{\mathbb{P}}^{\ell} \otimes \mathcal{O}_{X} \rightarrow 0
$$

Using the fact that $\mathrm{H}^{i}\left(\mathbb{P}, \Omega_{\mathbb{P}}^{j}(*)\right)=0$ for $1 \leq i \leq m, i \neq j$, we get isomorphisms

$$
\mathrm{H}^{n+\ell}\left(X, \Omega_{X}^{n-\ell}(-t)\right) \cong \mathrm{H}^{n+\ell+1}\left(X, \Omega_{X}^{n-\ell-1}(-t-d)\right) \forall 1 \leq \ell<n
$$

This finishes the proof.

Lemma 5. Let $X \subset \mathbb{P}^{m+1}$ be a smooth hypersurface with $m=2 n$. Then the map $\phi: \mathrm{H}^{n+1}\left(X, \Omega_{X}^{n-1}(-d)\right) \rightarrow V^{*} \otimes \mathrm{H}^{n+1}\left(X, \Omega_{X}^{n-1}\right)$ is injective for $d \geq 2+\frac{2}{n}$.

Proof. The map $\phi$ is induced by the composite map of sheaves $\Omega_{X}^{n-1} \otimes \mathcal{O}_{X}(-d)=$ $\Omega_{X}^{n-1}(-d) \rightarrow \Omega(n) \rightarrow \Omega_{X}^{n-1} \otimes V^{*}$ obtained from diagram (2.2) and the filtration with $\ell=n$. This map is clearly $1 \otimes-\beta$. Using the identifications in the previous lemma, we can identify the map $\phi$ with the dual of the cup product map

$$
\mathrm{H}^{2 n}\left(X, \mathcal{O}_{X}(-n d)\right) \rightarrow V^{*} \otimes \mathrm{H}^{2 n}\left(X, \mathcal{O}_{X}(-(n-1) d)\right.
$$

The lemma now follows by noting that the cup product map

$$
V \otimes \mathrm{H}^{0}\left(\mathcal{O}_{X}(n d-2 n-2)\right) \rightarrow \mathrm{H}^{0}\left(\mathcal{O}_{X}(n d+d-2 n-2)\right)
$$

is surjective as soon as $\mathrm{H}^{0}\left(\mathcal{O}_{X}(n d-2 n-2)\right) \neq 0$ which happens for all $d \geq 2+\frac{2}{n}$.

Proposition 1. Let $X \subset \mathbb{P}^{m+1}$ with $m=2 n$. Then

1) $\mathrm{H}^{p}\left(\mathbb{P}, \Omega_{\mathbb{P}}^{p}\right) \cong \mathrm{H}^{p}\left(X, \Omega_{X}^{p}\right) 0 \leq p \leq \operatorname{dim} X, p \neq n$

2) If $d \geq 3$, we also have

$$
\mathrm{H}^{n}\left(X, \Omega_{\mathbb{P}}^{n} \otimes \mathcal{O}_{X}\right) \cong \mathrm{H}^{n}\left(X, \Omega_{X_{\epsilon}}^{n} \otimes \mathcal{O}_{X}\right)
$$

Proof. The proof of the first part follows from the Weak Lefschetz theorem as in Lemma 2. For the second part, we consider the cohomology diagram associated to (2.2) when $\ell=n$.

$$
\begin{aligned}
& \mathrm{H}^{n}\left(X, \Omega_{X}^{n-1}(-d)\right) \rightarrow \mathrm{H}^{n}\left(X,\left.\Omega_{\mathbb{P}}^{n}\right|_{X}\right) \rightarrow \mathrm{H}^{n}\left(X, \Omega_{X}^{n}\right) \rightarrow \mathrm{H}^{n+1}\left(X, \Omega_{X}^{n-1}(-d)\right) \\
& \mathrm{H}^{n}(X, \Omega(n)) \rightarrow \mathrm{H}^{n}\left(X,\left.\Omega_{X_{\epsilon}}^{n}\right|_{X}\right) \rightarrow \mathrm{H}^{n}\left(X, \Omega_{X}^{n}\right) \rightarrow \mathrm{H}^{n+1}(X, \Omega(n))
\end{aligned}
$$

By Lemma 3, we have $\mathrm{H}^{n}(X, \Omega(n))=0$. Furthermore, by Kodaira-Akizuki-Nakano theorem (see [1], page 154) $\mathrm{H}^{n}\left(X, \Omega_{X}^{n-1}(-d)\right)=0$. This implies that $\mathrm{H}^{n}\left(X, \Omega_{\mathbb{P}}^{n} \otimes\right.$ $\left.\mathcal{O}_{X}\right) \rightarrow \mathrm{H}^{n}\left(X, \Omega_{X_{\epsilon}}^{n} \otimes \mathcal{O}_{X}\right)$ is injective. To prove surjectivity, it is enough to prove that the right most vertical arrow i.e. the map $\mathrm{H}^{n+1}\left(X, \Omega_{X}^{n-1}(-d)\right) \rightarrow \mathrm{H}^{n+1}(X, \Omega(n))$ is injective. By Lemma 5 , the composite $\mathrm{H}^{n+1}\left(X, \Omega_{X}^{n-1}(-d)\right) \rightarrow \mathrm{H}^{n+1}(X, \Omega(n)) \rightarrow$ $V^{*} \otimes \mathrm{H}^{n+1}\left(X, \Omega_{X}^{n-1}\right)$ is injective. Thus we are done.

Corollary 1. With notation as above,

$$
\text { Image }\left(\mathrm{H}^{i}\left(\mathbb{P}, \Omega_{\mathbb{P}}^{i}\right) \rightarrow \mathrm{H}^{i}\left(X, \Omega_{X}^{i}\right)\right)=\text { Image }\left(\mathrm{H}^{i}\left(X_{\epsilon}, \Omega_{X_{\epsilon}}^{i}\right) \rightarrow \mathrm{H}^{i}\left(X, \Omega_{X}^{i}\right)\right) \text {. }
$$


Proof. When $X$ is odd-dimensional, this follows from Lemma 2. When $\operatorname{dim} X=2 n$ and $i \neq n$, it follows from the first part of Proposition 1 . When $i=n$, this follows from the second part of the same proposition by noting that there is a factorisation $\mathrm{H}^{n}\left(X_{\epsilon}, \Omega_{X_{\epsilon}}^{n}\right) \rightarrow \mathrm{H}^{n}\left(X, \Omega_{X_{\epsilon}}^{n} \otimes \mathcal{O}_{X}\right) \rightarrow \mathrm{H}^{n}\left(X, \Omega_{X}^{n}\right)$.

\section{Vector bundles on hypersurfaces}

We start this section with a Lemma due to Faltings whose proof we omit.

Lemma 6 (Faltings, [2]). Let $\left(V, \mathcal{O}_{V}(1)\right)$ be any quasi-projective scheme. For an exact sequence of vector bundles

$$
0 \rightarrow G \rightarrow \oplus_{i=1}^{r} \mathcal{O}_{V}\left(a_{i}\right) \rightarrow E \rightarrow 0
$$

and for some $a=a_{i}$, let $G \stackrel{t_{1}}{\longrightarrow} \mathcal{O}_{V}(a)$ and $\mathcal{O}_{V}(a) \stackrel{t_{2}}{\longrightarrow} E$ denote the induced maps. Let $T_{1}$ and $T_{2}$ denote the zero schemes of $t_{1}$ and $t_{2}$ respectively. Then $T_{1} \cap T_{2}=\emptyset$.

We now prove the first two parts of Theorem 2.

Proof of Theorem 2 (1). The proof is by induction on $r$. The base case $r=1$ is trivial. Suppose that $E$ is an $r$-generated bundle. Then in the notation of Lemma 6 , if $E$ has rank $n$ and $G$ has rank $g$, then the dimensions of $T_{1}$ and $T_{2}$ are at least $m-g$ and $m-n$ respectively. Since their sum is at least $2 m-r \geq m+1, T_{1}$ and $T_{2}$ must intersect in projective space. Thus we arrive at a contradiction unless one of $T_{1}$ or $T_{2}$ is empty. If $T_{2}$ is empty, we may replace $E$ by a quotient $E^{\prime}$ which is $r-1$-generated. By induction, $E^{\prime}$ is a sum of line bundles and hence so is $E$. If we assume $T_{1}$ is empty, then by looking at the dual sequence, $G^{\vee}$ is split likewise and hence $G$ is split with $\mathcal{O}_{X}(a)$ as one of the summands. This implies that the map $\mathcal{O}_{X}(a) \rightarrow E$ is the zero map and hence $E$ is $r-1$-generated and hence split.

Proof of Theorem 2 (2). Suppose $E$ is $m$-generated. Using Lemma 6 , we get $T_{1}$ and $T_{2}$. If $T_{2}$ is empty, we would get a quotient which is $m-1$-generated. By part (1), this quotient and hence $E$ is split. If $T_{1}$ is empty, then a similar argument as above shows that $E$ is split. We claim that $T_{1}$ and $T_{2}$ have dimensions exactly equal to $m-g$ and $m-n$ respectively. For, if not, then $T_{1}$ and $T_{2}$ would have non-empty intersection in projective space, a contradiction.

Let $\left[T_{1}\right] \in \mathrm{H}^{g}\left(X, \Omega_{X}^{g}\right)$ and $\left[T_{2}\right] \in \mathrm{H}^{n}\left(X, \Omega_{X}^{n}\right)$ denote the Hodge classes of $T_{1}$ and $T_{2}$ respectively. Since neither $n$ nor $g$ is $m / 2$, using Lemma 2 and Proposition 1.1, we may assume that $\left[T_{1}\right]=\alpha h^{g}$ and $\left[T_{2}\right]=\beta h^{n}$ where $h$ is the class of the hyperplane section and $\alpha, \beta$ are non-zero scalars. Since $g+n=m$, this implies that $\left[T_{1}\right] \cdot\left[T_{2}\right]=\alpha \cdot \beta h^{m} \in$ $\mathrm{H}^{m}\left(X, \Omega_{X}^{m}\right)$ and hence non-zero. This contradicts the fact that $T_{1} \cap T_{2}=\emptyset$.

Lemma 7. Let $Y$ be a smooth projective variety and $E$ be a vector bundle of rank $n$ on $Y$. Let $s: \mathcal{O}_{Y} \rightarrow E$ be a morphism of sheaves such that the zero scheme $Z=Z(s)$ has codimension $n$ in $Y$. Then $\mathrm{c}_{n}(E)=0$ in $\mathrm{H}^{n}\left(Y, \Omega_{Y}^{n}\right)$ if and only $Z$ is empty.

Proof. If $Z$ is empty, then we have an exact sequence of bundles $0 \rightarrow \mathcal{O}_{Y} \stackrel{s}{\rightarrow} E \rightarrow$ $E^{\prime} \rightarrow 0$ and the result follows by the Whitney sum formula. For the converse, by taking general hyperplane sections, we may reduce to the case where $Z$ is a bunch of points in $Y$. It is a standard result that $c_{n}(E)=\operatorname{deg} Z$ (see [1], page 413). Since $\operatorname{deg} Z$ is the number of points in $Z$ counted with multiplicities, we are done. 
Proof of Theorem 2 (3). Let $X \subset \mathbb{P}^{m+1}, m=2 n$, be a smooth hypersurface of degree $d$ and let $E$ be a vector bundle of rank $n$ with $m$-generators so that it has a presentation

$$
\oplus_{i=1}^{s} \mathcal{O}_{X}\left(b_{i}\right) \rightarrow \oplus_{j=1}^{m} \mathcal{O}_{X}\left(a_{j}\right) \rightarrow E \rightarrow 0 .
$$

Let $S_{\underline{a}, \underline{b}} \subset S$ be the subset parametrising hypersurfaces which support non-split vector bundles $E$ with a presentation as above. We shall show that its closure $\bar{S}_{a, b}$ is a proper subset of $S$. Since there are only countably many choices for $\underline{a}, \underline{b}$, this will prove theorem 2.3.

By the arguments of theorem (3.4) in [6], there exists a scheme $\mathcal{P}^{\prime}(\underline{a}, \underline{b})$ along with a morphism $\mathcal{P}^{\prime}(\underline{a}, \underline{b}) \rightarrow S$ and a rank $n$ vector bundle on $\mathcal{X} \times{ }_{S} \mathcal{P}^{\prime}(\underline{a}, \underline{b})$ with a presentation as above. Furthermore, this scheme parametrises all such vector bundles, split or non-split.

Suppose $\mathcal{P}$ is a closed irreducible subvariety of $\mathcal{P}^{\prime}(a, b)$ which dominates $S$. By choosing a Zariski open subset of $\mathcal{P}$, we may assume that $\mathcal{P} \rightarrow S$ is smooth. We will show that for every point $p \in \mathcal{P}$ the corresponding bundle is split, proving the theorem.

Let $p \in \mathcal{P}$ be a point lying over $s \in S$. We can find a $\mathcal{P}^{\prime}$ such that $p \in \mathcal{P}^{\prime} \subset \mathcal{P}$ and $\mathcal{P}^{\prime} \rightarrow S$ is étale at $p$. Let $X:=X_{p}$ be the hypersurface parametrised by $p$ (and hence by $s$ ). Let $X_{\epsilon}$ be the universal hypersurface over $\operatorname{Spec} A$ where $A=\mathcal{O}_{\mathcal{P}^{\prime}, p} / \mathfrak{m}_{p}^{2}=$ $\mathcal{O}_{S, s} / \mathfrak{m}_{s}^{2}$. There is a rank $n$ bundle $\mathcal{E}$ on $X_{\epsilon}$ whose restriction to $X$ is a bundle $E$ (both are $m$-generated). We shall now show that $E$ is split.

Suppose $E$ is not split. Since $\mathcal{E}$ is $m$-generated, we have a short exact sequence:

$$
0 \rightarrow \mathcal{G} \rightarrow \oplus_{i=1}^{m} \mathcal{O}_{X_{\epsilon}}\left(a_{i}\right) \rightarrow \mathcal{E} \rightarrow 0
$$

where $\mathcal{G}$ is a vector bundle whose restriction to $X$ shall be denoted by $G$. Twisting the above sequence by a line bundle if necessary, we may assume that one of the $a_{j}$ 's is zero giving the diagram

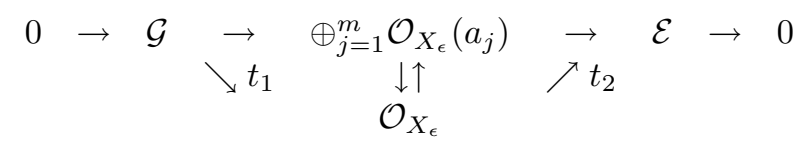

Let $\mathcal{Z}_{1}$ and $\mathcal{Z}_{2}$ denote the subschemes defined by the morphisms $t_{1}$ and $t_{2}$ respectively and $Z_{1}, Z_{2}$ be the zero schemes of the restrictions of the sections $t_{1}$ and $t_{2}$ respectively to $X$. On restricting the exact sequence to $X$, we have $Z_{1} \cap Z_{2}=\emptyset$ by Lemma 6 . As in the proof of Theorem 2.2, we may assume that $Z_{1}$ and $Z_{2}$ are non-empty with $\operatorname{codim} Z_{1}=n=\operatorname{codim} Z_{2}$.

We shall now use the theory of Chern classes for bundles on schemes with values in Hodge cohomology as described in [4] (see also [5] for a simpler exposition). Since one of the $a_{i}$ 's is zero, the Whitney sum formula for the exact sequence in (3.1), yields $\mathrm{c}_{n}(\mathcal{G}) \cdot \mathrm{c}_{n}(\mathcal{E})=0$. By functoriality, we have $\iota^{*} \mathrm{c}_{i}(\mathcal{G})=\mathrm{c}_{i}(G)$ and $\iota^{*} \mathrm{c}_{i}(\mathcal{E})=\mathrm{c}_{i}(E)$ for all $i$. Thus by Corollary $1, \mathrm{c}_{i}(G)$ and $\mathrm{c}_{i}(E)$ are in the one dimensional vector space Image $\left(\mathrm{H}^{i}\left(\mathbb{P}, \Omega_{\mathbb{P}}^{i}\right) \rightarrow \mathrm{H}^{i}\left(X, \Omega_{X}^{i}\right)\right) \forall i$. Putting these together, we have

$$
\mathrm{c}_{n}(G) \mathrm{c}_{n}(E)=i^{*} \mathrm{c}_{n}(\mathcal{G}) i^{*} \mathrm{c}_{n}(\mathcal{E})=i^{*}\left(\mathrm{c}_{n}(\mathcal{G}) \cdot \mathrm{c}_{n}(\mathcal{E})\right)=0
$$

and as a result, either $\mathrm{c}_{n}(G)=0$ or $\mathrm{c}_{n}(E)=0$. Since $Z_{1}$ and $Z_{2}$ have the correct codimension, $\mathrm{c}_{n}(G)=0$ (resp. $\left.\mathrm{c}_{n}(E)=0\right)$ if and only $Z_{1}=\emptyset\left(\right.$ resp. $\left.Z_{2}=\emptyset\right)$ by Lemma 7. This gives a contradiction. 
This proves that for a hypersurface which corresponds to a generic point (i.e. outside the countable union of the proper closed subvarieties $\left.\bar{S}_{\underline{a}, \underline{b}}\right)$ there are no $m$ generated, rank $n$ bundles which are not sums of line bundles.

\section{Acknowledgements}

This work was done when the third named author was a Chauvenet Lecturer in the Mathematics Department at Washington University in St. Louis. He would like to thank all the members of the department for their support, encouragement and for a wonderful working atmosphere.

\section{References}

[1] Griffiths, Phillip; Harris, Joseph., Principles of algebraic geometry, Wiley Classics Library. John Wiley and Sons, Inc., New York, 1994.

[2] Faltings, Gerd, Ein Kriterium für vollständige Durchschnitte, Invent. Math. 62 (1981), no. 3, 393-401.

[3] Hartshorne, Robin, Algebraic geometry, Graduate Texts in Mathematics, No. 52. Springer-Verlag, 1977.

[4] Illusie, Luc, Complexe cotangent et déformations. I, II, Lecture Notes in Mathematics, Vols. 239 and 283. Springer-Verlag.

[5] N. Mohan Kumar, A.P.Rao and G.V.Ravindra, Hodge style Chern classes for vector bundles on schemes, unpublished notes.

[6] _ Arithmetically Cohen-Macaulay bundles on hypersurfaces, Commentarii Math Helvetici (to appear).

[7] N. Mohan Kumar and V. Srinivas, The Noether-Lefschetz theorem, unpublished preprint.

Department of Mathematics, Washington University in St. Louis, St. Louis, Missouri, 63130

E-mail address: kumar@wustl.edu

$U R L:$ http://www.math. wustl.edu/ kumar

Department of Mathematics, University of Missouri-St. Louis, St. Louis, Missouri 63121

E-mail address: rao@arch.umsl.edu

Department of Mathematics, Indian Institute of Science, Bangalore-560 012, INDiA

E-mail address: ravindra@math.iisc.ernet.in 